\title{
On cognitive channels with an oblivion constraint
}

\author{
Daniela Tuninetti, Natasha Devroye, and Yasaman Keshtkarjahromi \\ University of Illinois at Chicago, Chicago IL \\ danielat, devroye, ykesht2@uic.edu
}

\begin{abstract}
We consider a Gaussian point-to-point secondary link which co-exists with a Gaussian point-to-point primary link, and is permitted to transmit within the primary user's interference margin - a simple but realistic channel model for underlay cognitive systems. Under this assumption, we are interested in quantifying the secondary user rate gains when the secondary users have knowledge of the primary link's codebook. This may be exploited at the secondary transmitter or receiver - we focus on the latter. As first proposed by Popovski et al., this codebook knowledge may be exploited to opportunistically - when channel conditions permit - either fully cancel the primary user interference at the secondary receiver, or otherwise treat it as noise. In this work, we propose an achievable rate region in which the primary user transmits according to an information theoretic broadcast strategy, but otherwise remains oblivious to the secondary user operation. The secondary receiver exploits the primary codebooks, together with its broadcast strategy transmission to decode a portion of the primary message, thereby opportunistically partially canceling the primary user interference seen at the secondary receiver and thus straddles the two extremes between fully decoding and treating the interference as noise as presented by Popovski et al. Interestingly, it is shown that this broadcast strategy does not outperform Popovski et al.'s simple "all or nothing" strategy. We thus seek to tighten outer bounds on the secondary user's channel capacity. The inclusion of an average power constraint on the secondary link furthermore allows us to develop a new, tighter outer bound for this channel, which is numerically evaluated and compared to the inner bounds.
\end{abstract}

Index Terms - cognitive network, broadcast strategy

\section{INTRODUCTION}

\section{A. Motivation}

Primary and secondary or cognitive users may co-exist in a number of ways [1], all with the goal of improving spectral efficiency: secondary signals may be 1) underlain with primary signals, meaning that they cause an acceptable and controlled amount of interference at primary receivers, or may alternatively be 2) overlain with primary signals, in which case the interference caused at primary receivers may be partially mitigated through secondary transmitter encoding schemes which exploit knowledge of the primary message [2], [3], or finally may be 3) interweaved with primary signals, in which case the the primary and secondary signals remain orthogonal. All schemes have merits and drawbacks; we will consider the first underlay paradigm regime for interfering Gaussian primary and secondary channels.

In the underlay regime, the primary user is assumed to operate with a built-in interference-margin. That is, it transmits at a rate strictly below the primary channel capacity in

* The work of D. Tuninetti and N. Devroye was partially supported by order to have some margin to protect itself from outages should unexpected noise / interference occur. In this case, secondary transmitters may transmit subject to power constraints dictated by the primary's interference-margin (more rigorously defined later). In contrast to previously considered information theoretic cognitive channels as in [2], [3], [1], we do not assume the secondary transmitter has knowledge of the primary user's message. Rather, we assume that the secondary link has knowledge of the primary user's codebook, and focus on how this knowledge may be exploited at the secondary receiver. In particular, as first outlined in [4], this codebook knowledge may allow - under certain channel conditions in which the primary signal is received at a high enough SNR at the secondary receiver - the cognitive receiver to decode the primary's message (which is assumed to be transmitted at constant, known rate), and subtract it off its received signal, cleaning the channel for its own transmission. Moreover, the primary link may be completely oblivious to this; all the burden is placed at the cognitive receiver. This "opportunistic interference cancellation" may thus enhance the performance of simple interference-temperature / margin based scheme at the cost of additional secondary receiver decoding complexity and willingness of primary users to make their codebooks available. We note that security is not necessarily compromised through codebook sharing, as cypher text may be used as data.

\section{B. Contributions}

In previous work, the secondary receiver either decoded the entire primary user's message if channel conditions would permit, or treated it entirely as noise if they did not. These are two extreme possibilities - one would expect the cognitive receiver to be able to decode whatever portion of the primary user signal its channel allows for, subtract this off the received signal, thereby partially cleaning the channel from its own transmitter. The central contributions of this paper are:

1) development of a time-sharing scheme at the secondary user which time-shares between the two extremes - treating the primary interference as noise and fully decoding it.

2) development of a primary encoding scheme which is independent and oblivious to the secondary user operation, and which allows the secondary receiver to jointly decode the optimal, with respect to the secondary rate, portion of the primary signal, thereby partially canceling interference at the secondary user. This scheme is derived from the information theoretic broadcast approach [5], useful for communication over fading channels whose fading state is unknown to the transmitter. Here all channel coefficients are known, but the primary user does not know (or wishes to remain oblivious) 
to the primary transmitter - secondary receiver channel. We furthermore demonstrate the surprising result that neither the time-sharing scheme nor the broadcast strategy scheme improves upon Popovski et al.'s simple yet powerful scheme which either treats interference as noise or fully decodes it [4].

3) development of an outer bound for this channel model tighter than existing ones. An immediate and obvious outer bound to the secondary user rate is that of an interferencefree channel as proposed in [4]. We improve upon this outer bound through the use of an outer bound originally derived for the two-user interference channel [6]. which is optimal "to within one bit" for the Gaussian channel.

4) numerical evaluation of the new inner and outer bounds in Gaussian noise which still indicate a gap to capacity. We comment on possible directions to close this gap.

\section{Outline}

We formally define the channel model and assumptions in Section II, before proposing a new achievable secondary rate with exploits opportunistic (partial) interference cancelation with a primary broadcast strategy in Section III. We present the new outer bound based on [6] in Section IV, numerically compare inner and outer bounds in Gaussian noise in Section $\mathrm{V}$, and conclude in Section VI.

\section{PRoblem DEFinition, NOTATION}

\section{A. General Model}

We consider two point-to-point channels, one primary transmitter-receiver (Tx-Rx) pair indexed by subscript 0 , and another secondary or cognitive Tx-Rx pair indexed by subscript 1 . These two channels co-exist and interfere with each other. We assume a classic information theoretic framework and we adopt classical definitions for achievable rates and capacity. In particular, we assume that the primary user is transmitting at a fixed rate $R_{0}=r_{0}$ and we say that a rate $R_{1}$ is achievable for the secondary link if its error probability can be made arbitrarily small. The capacity of the secondary link is the supremum of all achievable secondary rates.

\section{B. Additive White Gaussian Noise (AWGN) channel}

We are interested in the AWGN channel model depicted in Fig. 1 and described, at each channel use, by:

$Y_{0}=h_{00} X_{0}+h_{01} X_{1}+Z_{0}, Z_{0} \sim \mathcal{N}\left(0, N_{0}\right), \mathbb{E}\left[\left|X_{0}\right|^{2}\right] \leq \bar{P}_{0}$, $Y_{1}=h_{10} X_{0}+h_{11} X_{1}+Z_{1}, Z_{1} \sim \mathcal{N}\left(0, N_{1}\right), \mathbb{E}\left[\left|X_{1}\right|^{2}\right] \leq \bar{P}_{1}$,

where without loss of generality we assume:

$$
\bar{P}_{0}=\bar{P}_{1}=N_{1}=1, N_{0}=1+I_{0}, I_{0} \geq 0 .
$$

The term $I_{0}$ is the instantaneous interference margin at the primary receiver. That is, the primary user is assumed to transmit at a rate $r_{0}$ bits per channel use and can tolerate interference of power up to $I_{\max }$ (this is similar to "interference temperature" [7]). If the actual interference at the primary receiver is of power $I_{\text {actual }}$ then we call $I_{0}=I_{\max }-I_{\text {actual }}$ the interference margin at the primary receiver.

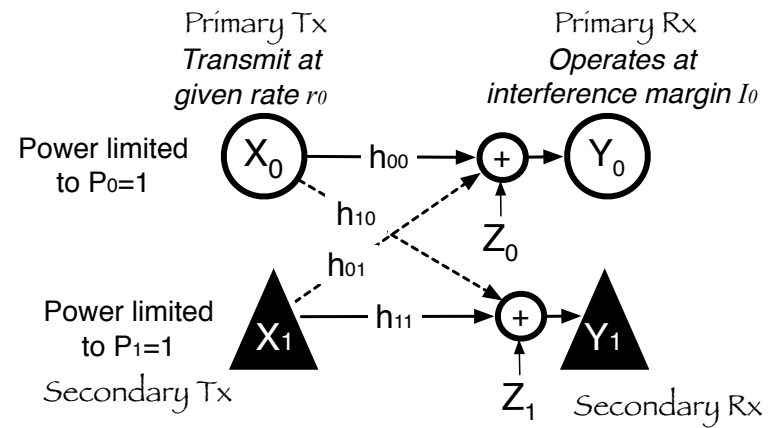

Fig. 1. The Gaussian channel considered.

\section{Assumptions}

In this channel model, we wish to emphasize a number of assumptions/restrictions we place on the channel, which are motivated by future cognitive networks:

1. Primary $\mathbf{T x} / \mathbf{R x}$ : we assume that the primary user transmits using a Gaussian codebook at a fixed rate $r_{0}$ subject to a unit power constraint as

$$
r_{0}=\log \left(1+\frac{\left|h_{00}\right|^{2}}{1+I_{0}}\right),
$$

and the primary receiver treats $X_{1}$ as noise. We will propose a brodcast strategy-inspired codebook for the primary user. We only permit the secondary user to transmit as long as the primary rate remains constant at $r_{0}$.

2. Primary remains oblivious: we assume that the primary does not change its encoder or decoder, or set any of their parameters as a function of the secondary user operation.

3. Non-trivial interference margin: we assume $I_{0}>0$ so as to allow the secondary user to transmit. The power of the secondary user has to be such that the "instantaneous" interference created at the primary receiver is within the interference margin. We will propose a Time Division Multiplexing strategy for the secondary transmitter and will impose that in each transmission phase the interference margin is respected.

If we relax the "instantaneous" interference margin constraint to an average one, then the power constraint for the secondary user is $p^{*}=\min \left\{1, I_{0} /\left|h_{01}\right|^{2}\right\}$. We will derive an outer bound for the proposed system by considering this modified power constraint for the secondary user.

4. Codebook and channel knowledge: we assume that the secondary receiver has the primary user's codebook, and that the secondary Tx and Rx both have knowledge of all channel gains $h_{i j}, i, j \in\{0,1\}$.

The goal of this paper is to derive the optimal, in terms of secondary user capacity, scheme subject to these various cognitive and oblivion constraints.

\section{INNER BOUNDS}

This problem, with all constraints except for the average power constraint on the secondary user $\mathbb{E}\left[\left|X_{1}\right|^{2}\right] \leq \bar{P}_{1}=$ 1 was first posed by Popovski et al. in [4]. We outline the simple yet powerful scheme proposed there, but add on the extra power constraint $\mathbb{E}\left[\left|X_{1}\right|^{2}\right] \leq \bar{P}_{1}=1$ so as to allow for comparison with our proposed scheme. 


\section{A. Known inner bound [4]}

The achievable rate for the secondary user's rate $R_{1}$ was derived as follows in [4]:

$$
R_{1, \mathrm{pop}}=\max \left\{R_{1, \mathrm{ni}}\left(p^{*}\right), R_{1, \mathrm{jd}}\left(p^{*}\right)\right\},
$$

where we recall $p^{*}=\min \left\{1, I_{0} /\left|h_{01}\right|^{2}\right\}$ and where we defined

$$
\begin{aligned}
R_{1, \mathrm{ni}}(p) & =\sup \left\{R_{1} \geq 0: R_{1} \leq \log \left(1+\frac{\left|h_{11}\right|^{2} p}{1+\left|h_{10}\right|^{2}}\right)\right\} \\
& =\log \left(1+\left|h_{11}\right|^{2} \frac{p}{1+\left|h_{10}\right|^{2}}\right)
\end{aligned}
$$

and

$$
\begin{gathered}
R_{1, \mathrm{jd}}(p)=\sup \left\{R_{1} \geq 0: R_{1} \leq \log \left(1+\left|h_{11}\right|^{2} p\right),\right. \\
\left.R_{1}+r_{0} \leq \log \left(1+\left|h_{11}\right|^{2} p+\left|h_{10}\right|^{2}\right)\right\} \\
=\min \left\{\log \left(1+\left|h_{11}\right|^{2} p+\left|h_{10}\right|^{2}\right)-r_{0}, \log \left(1+\left|h_{11}\right|^{2} p\right)\right\} .
\end{gathered}
$$

Remark: we note that the rate $R_{1, \mathrm{jd}}(p)$ is described differently in [4]. By seeing the primary and secondary users as forming a Multiple Access Channel (MAC) at the secondary Rx, the author in [4] includes the rate constraint $r_{0} \leq \log \left(1+\left|h_{10}\right|^{2}\right)$ in the achievable region. However, since the secondary user is not interested in the message of the primary user, the rate constraint $r_{0} \leq \log \left(1+\left|h_{10}\right|^{2}\right)$ is not necessary to drive the secondary error probability to zero and can thus be omitted. Despite this difference, the achievable rate that results from choosing the best strategy between joint decoding and treating interference as noise is the same with both descriptions.

\section{B. Proposed inner bound: Time Division Multiplexing (TDM)}

One may ask whether an intermediate strategy between treating the primary signal entirely as noise, or decoding it entirely may outperform the extreme cases. One such intermediate strategy that the secondary Tx-Rx link may employ is a simple TDM strategy, where we time share between the rates achieved by 'ni' = "Gaussian coding and noisy interference" and 'jd' = "Gaussian coding and joint decoding", the two extremes considered in [4]. Our proposed rate is thus $R_{, t d m}$ :

$$
R_{1, \mathrm{tdm}}=\max _{\left(\tau, P_{a}, P_{b}\right) \in \mathcal{D}}\left\{\tau R_{1, \mathrm{ni}}\left(P_{a}\right)+(1-\tau) R_{1, \mathrm{jd}}\left(P_{b}\right)\right\},
$$

where the maximization domain $\mathcal{D}$ is

$$
\begin{aligned}
\mathcal{D}= & \left\{\tau \in[0,1], P_{a} \geq 0, P_{b} \geq 0,\right. \\
& \left.\tau P_{a}+(1-\tau) P_{b} \leq 1,\left|h_{01}\right|^{2} P_{a} \leq I_{0},\left|h_{01}\right|^{2} P_{b} \leq I_{0}\right\} .
\end{aligned}
$$

Clearly $R_{1, \mathrm{tdm}} \geq R_{1, \mathrm{pop}}$, as $R_{1 \text {,pop }}$ coincides with the max between $\tau=0$ and $\tau=1$. However, we have:

Proposition 1: We have $R_{1, \mathrm{tdm}}=R_{1, \mathrm{pop}}$.

Proof: Time Division Multiplexing does not increase the MAC capacity region [8] and so cannot increase $R_{1, \text { pop }}$.

\section{Proposed inner bound: broadcast approach}

We now present an alternative scheme which interpolates between treating the primary interference as noise and fully decoding it at the secondary $\mathrm{Rx}$ based on the "broadcast approach" first proposed in the context of fading channels in [5], [9]. The "broadcast approach" consists of a transmission strategy in which many layers are superimposed at the transmitter and a portion or these layers (in the case of fading channels) are successively decoded at the receiver. This is particularly useful in scenarios where the transmitter does not know the fading experienced on the channel to its receiver, and allows the Rx to adaptively decode as much as it can, depending on the actual realization of the fade. We adapt this strategy for the primary user, who is to remain oblivious to the secondary user operation, forbidding rate-splitting techniques where the rate-split parameter would depend on the primary Tx - secondary Rx link condition.

We assume that the primary user uses a superposition of $L \in \mathbb{N}$ independent Gaussian codes; in the limit $L \rightarrow \infty$, this is known as the "broadcast approach". Let $\beta_{\ell} \in[0,1]$ $\forall \ell \in[1: L]$ and such that $\sum_{\ell=1}^{L} \beta_{\ell}=1$ and let the primary Tx send $X_{0}=\sum_{\ell=1}^{L} \sqrt{\beta_{\ell}} X_{0, \ell}$, where the $X_{0, \ell}$ are iid $\mathcal{N}(0,1)$ $\forall \ell \in[1: L]$. Each $X_{0, l}$ corresponds to the encoding of one layer carrying a primary message at a given rate $r_{l}$. Assume that the primary transmitter performs superposition coding in the order $X_{0,1} \rightarrow X_{0,2} \rightarrow \ldots \rightarrow X_{0, L}$ and that the primary receiver performs joint decoding of the $L$ layers by treating the secondary signal as noise. In the following we use the convention $\sum_{\ell=L+1}^{L} \beta_{\ell}=0$ and $\sum_{\ell=k}^{0} \beta_{\ell}=0$. The achievable rate for this primary transmission scheme is

$$
r_{0}=\sum_{\ell=1}^{L} r_{\ell}
$$

where the set $\left\{r_{\ell} \geq 0, \forall \ell \in[1: L]\right\}$ satisfies

$$
\sum_{\ell=k}^{L} r_{\ell} \leq \log \left(1+\frac{\left|h_{00}\right|^{2}}{1+I_{0}} \sum_{\ell=k}^{L} \beta_{\ell}\right), k \in[1: L] .
$$

The secondary receiver performs joint decoding of its own signal and the first $M, 0 \leq M \leq L$, layers of the primary user's signal by treating the rest of the layers as noise. The achievable rate with the broadcast approach is

$$
R_{1, \mathrm{bc}}=\max _{M \in[0, L]} R_{1, M}\left(p^{*}\right),
$$

with

$$
\begin{array}{r}
R_{1, M}(p)=\sup \left\{R_{1} \geq 0: \forall k \in[1: M]\right. \\
R_{1} \leq \log \left(1+\frac{\left|h_{11}\right|^{2} p}{1+\left|h_{10}\right|^{2} \sum_{\ell=M+1}^{L} \beta_{\ell}}\right), \\
\left.\sum_{\ell=k}^{M} r_{\ell}+R_{1} \leq \log \left(1+\frac{\left|h_{11}\right|^{2} p+\left|h_{10}\right|^{2} \sum_{\ell=k}^{M} \beta_{\ell}}{1+\left|h_{10}\right|^{2} \sum_{\ell=M+1}^{L} \beta_{\ell}}\right)\right\} .
\end{array}
$$

Remark: we note that this rate generalizes and contains $R_{1 \text {,pop }}$, as $M=0$ corresponds to "ni", while the case $M=L$ corresponds to "jd" as defined before.

We now consider the limit for an infinite number of layers, or $L \rightarrow \infty$. For a power distribution function $\beta(s) \geq 0, s \geq 0$, 
i.e., $\int_{0}^{\infty} \beta(s) \mathrm{d} s=1$, let $F(y)=\int_{0}^{y} \beta(s) \mathrm{d} s$ be the corresponding cumulative distribution function. The achievable rate with the broadcast approach is (intuitively correspond $k \rightarrow x$ and $M \rightarrow y$ with $0 \leq x<y$ )

$$
\begin{aligned}
R_{1, \mathrm{bc}}(p) & =\sup _{y \geq 0} \inf _{x \in[0, y)}\left[\log \left(1+\frac{\left|h_{11}\right|^{2} p+\left|h_{10}\right|^{2}(F(y)-F(x))}{1+\left|h_{10}\right|^{2}(1-F(y))}\right)\right. \\
& \left.-\log \left(1+\frac{\left|h_{00}\right|^{2}(F(y)-F(x))}{1+I_{0}+\left|h_{00}\right|^{2}(1-F(y))}\right)\right]^{+}
\end{aligned}
$$

since the incremental rate for the primary user is

$$
\begin{aligned}
r_{k} & =\log \left(1+\frac{\left|h_{00}\right|^{2}}{1+I_{0}} \sum_{\ell=k}^{L} \beta_{\ell}\right)-\log \left(1+\frac{\left|h_{00}\right|^{2}}{1+I_{0}} \sum_{\ell=k+1}^{L} \beta_{\ell}\right) \\
& =\frac{\left|h_{00}\right|^{2} \beta_{k}}{1+I_{0}+\left|h_{00}\right|^{2} \sum_{\ell=k}^{L} \beta_{\ell}}+o\left(\beta_{k}\right) \text { for } \beta_{k} \rightarrow 0 .
\end{aligned}
$$

Thus, recalling that $\beta(s) \mathrm{d} s=\mathrm{d} F(s)$,

$$
\begin{aligned}
\sum_{\ell=k}^{M} r_{\ell} & \rightarrow \int_{x}^{y} \frac{\left|h_{00}\right|^{2}}{1+I_{0}+\left|h_{00}\right|^{2}(1-F(s))} \mathrm{d} F(s) \\
& =\log \left(1+\frac{\left|h_{00}\right|^{2}(F(y)-F(x))}{1+I_{0}+\left|h_{00}\right|^{2}(1-F(y))}\right) .
\end{aligned}
$$

We now optimize the secondary rate given in (2) over $x, y$ corresponding intuitively to the fraction of primary layers decoded at the secondary $\mathrm{Rx}$, and show that the result is equal to the rate $R_{1, \text { pop }}$ achieved by the simpler scheme described in Section III-A.

Proposition 2: We have

$$
\begin{aligned}
& R_{1, \mathrm{bc}}(p)=\max _{\beta \in[0,1]} \min \left\{\log \left(1+\frac{\left|h_{11}\right|^{2} p}{1+\left|h_{10}\right|^{2}(1-\beta)}\right),\right. \\
& \log \left(1+\frac{\left|h_{11}\right|^{2} p+\left|h_{10}\right|^{2} \beta}{1+\left|h_{10}\right|^{2}(1-\beta)}\right) \\
& \left.-\log \left(1+\frac{\left|h_{00}\right|^{2} \beta}{1+I_{0}+\left|h_{00}\right|^{2}(1-\beta)}\right)\right\}=R_{1, \mathrm{pop} .}
\end{aligned}
$$

Proof: We first prove that the broadcast rate region in (2) is equal to (3), and then show that this is in turn maximized at either $\beta=0$ or $\beta=1$, in which case (3) is equal to (1).

The optimization over $x \in[0, y)$ is equivalent to the optimization over $t=F(y)-F(x) \in(0, F(y)]$ and may be carried out as follows: let $A=\frac{\left|h_{11}\right|^{2} p}{1+\left|h_{10}\right|^{2}(1-F(y))} \geq 0$, $B=\frac{\left|h_{10}\right|^{2}}{1+\left|h_{10}\right|^{2}(1-F(y))} \geq 0$, and $C=\frac{\left|h_{00}\right|^{2}}{1+I_{0}+\left|h_{00}\right|^{2}(1-F(y))} \geq 0$; the function $g(t)=\log (1+A+B t)-\log (1+C t)$ is either increasing or decreasing in $t \in \mathbb{R}^{+}$, thus $\arg \min \{g(t)\}$ is either $t=0$ or $t=F(y)$. By setting $F(y)=\beta \in[0,1]$ we obtain the claimed result.

Next, we note that taking $\beta=0$ we obtain the rate $R_{1, \mathrm{ni}}(p)$, and by taking $\beta=1$ we obtain the rate $R_{1, \mathrm{jd}}(p)$. Thus, the rate $R_{1, \text { pop }}$ is obtained by taking the maximum of our region over $\beta \in\{0,1\}$. Intuitively, one may expect some $\beta \in(0,1)$ to achieve a larger rate, but we now show that this is not that case, i.e., the rate achieved by the broadcast approach is maximized at $\beta \in\{0,1\}$. To do so, we first set $f_{1}(\beta)=$ $\log \left(1+\frac{\left|h_{11}\right|^{2} p}{1+\left|h_{10}\right|^{2}(1-\beta)}\right)$, and $f_{2}(\beta)=\log \left(1+\frac{\left|h_{11}\right|^{2} p+\left|h_{10}\right|^{2} \beta}{1+\left|h_{10}\right|^{2}(1-\beta)}\right)-$ $\log \left(1+\frac{\left|h_{00}\right|^{2} \beta}{1+I_{0}+\left|h_{00}\right|^{2}(1-\beta)}\right)$ and note that $f_{1}(0)=f_{2}(0)$. It is easy to see that $f_{1}(\beta)=f_{2}(\beta)$ only for $\beta=0$ since

$$
\begin{aligned}
2^{f_{1}}-2^{f_{2}} & =1+\frac{\left|h_{11}\right|^{2} p}{1+\left|h_{10}\right|^{2}(1-\beta)}-\frac{1+\frac{\left|h_{11}\right|^{2} p+\left|h_{10}\right|^{2} \beta}{1+\left|h_{10}\right|^{2}(1-\beta)}}{1+\frac{\left|h_{00}\right|^{2} \beta}{1+I_{0}+\left|h_{00}\right|^{2}(1-\beta)}} \\
& =\frac{\beta\left(\left|h_{00}\right|^{2}-\left|h_{10}\right|^{2}-I_{0}\left|h_{10}\right|^{2}+p\left|h_{00}\right|^{2}\left|h_{11}\right|^{2}\right)}{\left(1+\left|h_{10}\right|^{2}(1-\beta)\right)\left(1+I_{0}+\left|h_{00}\right|^{2}\right)} .
\end{aligned}
$$

Moreover, since both $f_{1}(\beta)$ and $f_{2}(\beta)$ are either monotonically increasing or monotonically decreasing in $\beta$, they only intersect at $\beta=0$. Lastly, $f_{1}$ and $f_{2}$ are each maximized at either $\beta=0$ or $\beta=1$ as they are both monotonic. Hence, the optimal value is either $\beta=0$ or $\beta=1$ thus implying that the proposed broadcast strategy for the primary user results in the same rate as $R_{1, \text { pop. }}$

Remark: This proposition states that "partial" interference cancelation of the primary transmission, which uses a broadcast strategy, at the secondary receiver does not improve the secondary rate over the simple scheme proposed in [4]. This has important implications in the design of cognitive radio systems, and may indicate that treating an undecodable signal as noise is optimal in Gaussian channels. This was observed, and proven to be true for a class of "good" Gaussian codebooks in [10]. Whether the results of [10] apply to this channel model and thus would give us a converse is the subject of ongoing work. The results of [10] are not immediately applicable as the primary user is operating at an interference margin (and thus below capacity) which may or may not fall under the "good" codebooks category for which the converse in $[10]$ is proven.

\section{OUTER BOUNDS}

We now present an existing and a new outer bound for the secondary user's rate.

\section{A. Known outer bound [4]}

Clearly, as observed in [4], the secondary user rate cannot exceed the interference-free capacity of a channel where the "instantaneous" interference margin constraint has been relaxed to an average one, that is,

$$
R_{1} \leq C_{1, \mathrm{tri}}=\log \left(1+\left|h_{11}\right|^{2} p^{*}\right),
$$

where we recall $p^{*}=\min \left\{1, I_{0} /\left|h_{01}\right|^{2}\right\}$.

\section{B. Proposed outer bound}

We obtain a tighter outer bound than $C_{1, \text { tri }}$ in (4) by relaxing the constraint that the primary transmitter and receiver are oblivious to the secondary user operation. That is, the capacity region of the channel considered in this work is contained in the information theoretic interference channel (IC) capacity. The following is thus an outer bound:

$$
R_{1} \leq C_{1, \text { etw }}=\sup \left\{R_{1} \geq 0:\left(R_{1}, r_{0}\right) \in \mathcal{C}_{e t w}\right\}
$$


where $\mathcal{C}_{\text {etw }}$ is the outer bound region on the Gaussian IC proposed by Etkin, Tse and Wang (ETW) [6] evaluated for

$$
\begin{aligned}
& \mathrm{SNR}_{0}=\left|h_{00}\right|^{2}, \mathrm{INR}_{1}=\left|h_{01}\right|^{2} p^{*}, \\
& \mathrm{INR}_{0}=\left|h_{10}\right|^{2}, \mathrm{SNR}_{1}=\left|h_{11}\right|^{2} p^{*} .
\end{aligned}
$$

Proposition 3: We have $C_{1 \text {,etw }} \leq C_{1, \text { tri }}$.

Proof: By relaxing the primary "oblivion" constraints we obtain a Gaussian IC. The result follows since $C_{1, \text { tri }}$ is only one of the constraints of the outer bound region $\mathcal{C}_{\text {etw }}$.

\section{NumericAl RESUlts}

Figs. 2 and 3 show the achievable rate $R_{1, \mathrm{pop}}=R_{1, \mathrm{tdm}}=$ $R_{1, \mathrm{bc}}$ and the outer bounds $C_{1, \text { tri }}$ and $C_{1, \text { etw }}$ as a function of the interference margin $I_{0}$ and of the secondary direct link gain $h_{11}$, respectively. The proposed outer bound $C_{1, \text { etw }}$ is clearly tighter than the previously known $C_{1, \text { tri }}$.

\section{CONCLUSION}

In this paper we considered inner and outer bounds on the capacity of a cognitive system where a primary transmitterreceiver pair operates at a fixed rate and is oblivious of any other activity the network, and a secondary transmitter-receiver can access the network subject to an interference margin constraint. We proposed two strategies to improve on the simple achievable scheme where the secondary receiver either treats the interference as noise or fully decodes it: time division multiplexing and opportunistic decoding of an infinite layer broadcast approach code. We showed - somehow surprisingly - that neither scheme improves over the achievable "all or nothing" strategy. We also proposed a novel outer bound that is tighter than an existing one. An interesting and challenging open question is whether the "all or nothing" strategy is actually optimal. In order to answer this question in the positive a more powerful outer bound seems to be needed.

\section{REFERENCES}

[1] A. Goldsmith, S. Jafar, I. Maric, and S. Srinivasa, "Breaking spectrum gridlock with cognitive radios: An information theoretic perspective," Proc. IEEE, vol. 97, no. 5, pp. 894-914, May 2009.

[2] N. Devroye, P. Mitran, and V. Tarokh, "Achievable rates in cognitive radio channels," IEEE Trans. Inf. Theory, vol. 52, no. 5, pp. 1813-1827, May 2006.

[3] A. Jovicic and P. Viswanath, "Cognitive radio: An information-theoretic perspective," Submitted to IEEE Trans. Inf. Theory, 2006.

[4] P. Popovski, H. Yomo, K. Nishimori, R. D. Taranto, and R. Prasad, "Opportunistic interference cancellation in cognitive radio systems," in Proc. 2nd IEEE International Symposium on Dynamic Spectrum Access Networks (DySPAN), Dublin, Ireland, Apr. 2007.

[5] S. Shamai and A. Steiner, "A broadcast approach for a single-user slowly fading MIMO channel," IEEE Trans. Inf. Theory, vol. 49, no. 10, pp. 2617-2635, 2003.

[6] R. Etkin, D. Tse, and H. Wang, "Gaussian interference channel capacity to within one bit," IEEE Trans. Inf. Theory, vol. 54, no. 12, pp. 55345562, Dec. 2008.

[7] P. Kolodzy, "Interference temperature:a metric for dynamic spectrum utilization," International Journal of Network Management, pp. 103113, Mar. 2006.

[8] T. Cover and J. Thomas, Elements of Information Theory, 2nd ed. New York: Wiley, 2006.

[9] S. Shamai, "A broadcast strategy for the Gaussian slowly fading channel," in Proc. IEEE Int. Symp. Inf. Theory. IEEE, 1997, p. 150.

[10] R. Tandra and A. Sahai, "Is interference like noise when you know its codebook?" in 2006 IEEE International Symposium on Information Theory, July 2006.

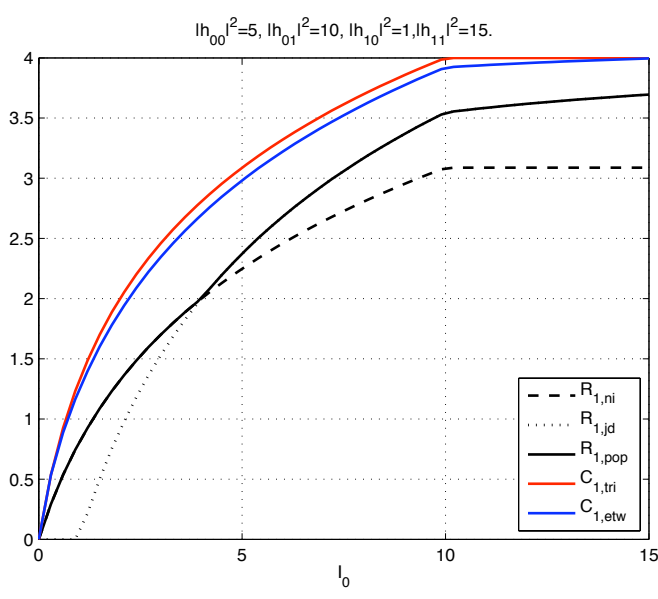

Fig. 2. Achievable rate and outer bounds vs the interference temperature $I_{0}$.

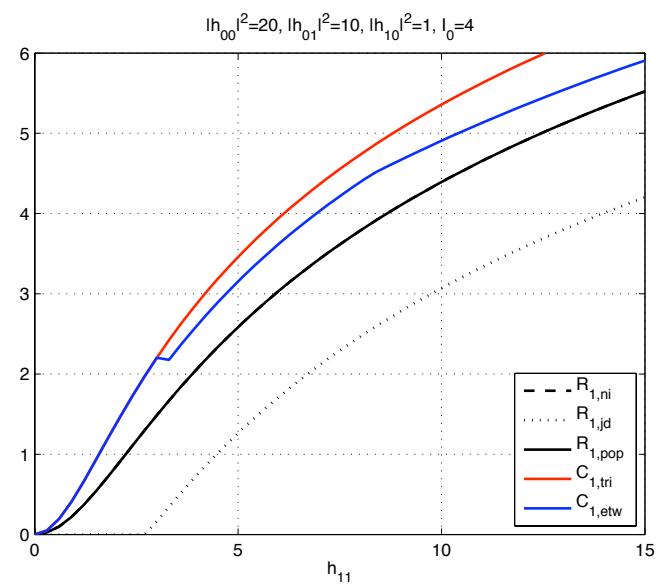

Fig. 3. Achievable rate and outer bounds vs the direct link gain of the secondary user $h_{11}$.

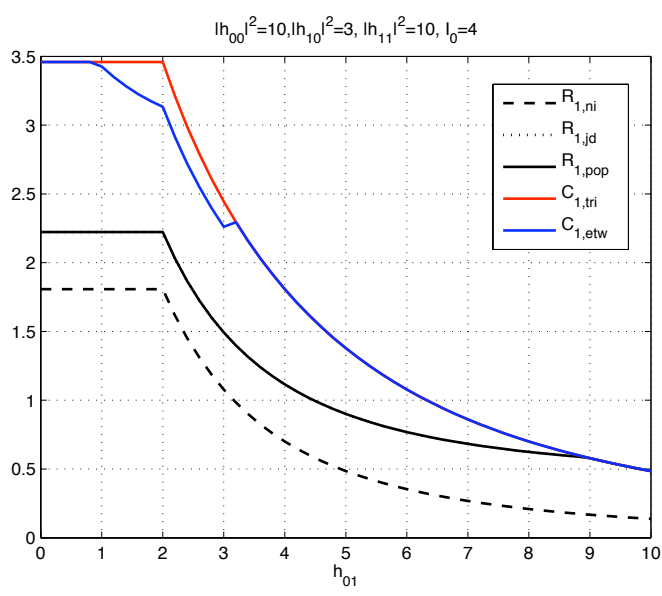

Fig. 4. Achievable rate and outer bounds vs the interference link gain of the primary user $h_{01}$. 